\title{
Uncooled thermocouple air-bridge structure for a THz imaging system
}

\author{
U. Schinkel, E. Kessler, A.lhring, U. Dillner, F. Haenschke, A. Brown, T. May, H.-G. Meyer \\ Institute for Photonic Technology e.V.(IPHT), Albert-Einstein-Str- 9, D-07745 Jena
}

\begin{abstract}
:
We have developed an air-bridge structure supporting a thermocouple-based conducting strip, which acts as both a terminating resistance of an antenna and temperature sensor [1]. The fabrication of the $30 \mu \mathrm{m}$ long and $3.5 \mu \mathrm{m}$ wide air-bridge was accomplished by surface micromachining with a polyimide sacrificial layer. As the supporting layer, a $200 \mathrm{~nm}$ PE-CVD Si $\mathrm{N}_{y}$ layer was patterned on the sacrificial layer. For passivation a $25 \mathrm{~nm}$ thin ALD-SiO ${ }_{2}$ with a low thermal conductance and a high conformal coverage was used. The influences of the geometry, the substrate thickness and the thermal properties of the freestanding air-bridges were investigated by computer simulations. Based on these results a compatible technology was developed to obtain high performance thermoelectric terahertz $(\mathrm{THz})$ sensing structures. The responsivity of the thermocouple air-bridge structure was evaluated by electrical characterization. For these measurements the sensing structures were loaded by a welldefined AC bias, which simulated an absorbed incoming terahertz radiation and its conversion into heat resulting in a corresponding measurable DC signal. The NEP of the structure was measured to be $18 \mathrm{pW} / \mathrm{Hz}^{1 / 2}$ under vacuum conditions and $67 \mathrm{pW} / \mathrm{Hz}^{1 / 2}$ under atmospheric conditions, respectively.
\end{abstract}

Key words: Surface micromachining, High detectivity, Antenna coupled, THz detectors

\section{Introduction}

In recent years, $\mathrm{THz}$ detectors have been investigated for applications in the fields of security screening, medicine gas sensing and food industry. For various types of THz detectors, antennas are an efficient way for coupling the signal to be detected. They can be used for cooled $[2,3]$ and uncooled $[1,4,5,6] \mathrm{THz}$ detection. In these sensors, the energy of the terahertz radiation is absorbed and dissipated by an appropriate planar antenna. Here, an airbridge can serve as thermal isolation structure, thus amplifying the resulting temperature increase. The temperature rise can be transduced into an electric signal voltage by the thermo resistive effect using a bolometer $[2,3,4,6]$ or by the Seebeck effect employing a thermocouple $[1,5]$. Typically, both Joule's heating and temperature sensing are enabled by one and the same conducting strip $[1,2,3,4,5]$ which should, therefore, be impedance-matched to the antenna.

\section{Thermal modeling of the air-bridge}

The thermal model calculates the temperature distribution due to heat load caused by radiation absorption. The considered bridge structure is shown in Fig. 1. Both the partial strips of antimony $(200 \mathrm{~nm})$ and bismuth antimony
$(350 \mathrm{~nm})$, with different electrical and thermal conductivity, have to be included for the examination of the bridge structure. The electrical resistance of this structure must be adjusted to the antenna impedance of 250-350 $\Omega$.

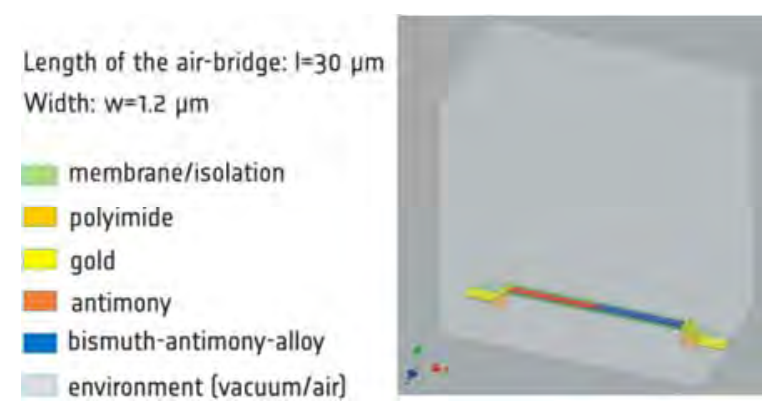

Figure 1: Solid model of a symmetric thermocouple structure taken as basis for the thermal simulation.

Thus, the geometry is mostly predefined. Taking into account the known resistivities of the used materials, the length and width of the air bridge becomes $30 \mu \mathrm{m}$ and $3.5 \mu \mathrm{m}$, respectively. Furthermore, a total thickness of $550 \mathrm{~nm}$ was assumed including the membrane and the passivation layer. Gold was used at both ends of the air bridge to serve as electrical connectors to the antenna as well as heat conductors to the substrate. The operating environment is air with a temperature of $300 \mathrm{~K}$, or vacuum. The FEM software COMSOL was used to calculate the 
Table 1: Results of the simulation for vacuum conditions.substrate.

\begin{tabular}{|l|l|}
\hline Physical parameter & \multicolumn{1}{|c|}{$\begin{array}{c}\text { Results of the } \\
\text { simulation }\end{array}$} \\
\hline Electrical resistivity & $270 \Omega$ \\
\hline $\begin{array}{l}\text { Thermal } \\
\text { conductance (vac.) }\end{array}$ & $1 \mu \mathrm{W} / \mathrm{K}$ \\
\hline Time constant (vac.) & $90 \mu \mathrm{s}$ \\
\hline Responsivity (vac.) & $140 \mathrm{~V} / \mathrm{W}$ \\
\hline NEP (vac.) & $16 \mathrm{pW} / \mathrm{Hz}^{1 / 2}$ \\
\hline Vacuum factor & 3.5 \\
\hline
\end{tabular}

temperature distribution in the thermocouple airbridge structure. The resulting stationary 2D temperature distribution is displayed in Fig. 2 for a power load of $7.5 \mu \mathrm{W}$. In air, the maximum temperature increase is $2.6 \mathrm{~K}$, whereas in vacuum it is $9 \mathrm{~K}$. This results in a vacuum factor (ratio between temperature rise in vacuum and air) of 3.5, cf. Tab 1. The thermal conductance G can be calculated as

$$
G=\frac{P}{\Delta T}
$$

where $\mathrm{P}$ is the power load and $\Delta \mathrm{T}$ the temperature difference between the hot and cold junction of the thermocouple. For vacuum, the thermal conductance is about $1 \mu \mathrm{W} / \mathrm{K}$. The responsivity is given by

$$
S=\frac{\gamma}{G[1+i \omega \tau)}
$$

with $\gamma=135 \mu \mathrm{V} / \mathrm{K}$ being the Seebeck-coefficient of the thermocouple, $\omega=2 \pi f$ the angular frequency and $\tau$ the thermal time constant. For $\omega=0$ the responsivity in vacuum is $140 \mathrm{~V} / \mathrm{W}$. The noise of the thermocouple is principally affected by two components: the Johnson noise and the thermal fluctuation noise. In all practical cases, however, the Johnson noise is dominating [7].

$$
N E P^{2}=N E P_{T}^{2}+N E P_{J}^{2}
$$

The NEP due to the thermal fluctuation noise is calculated by

$$
N E P_{T}^{2}=4 k_{B} T^{2} \cdot G
$$

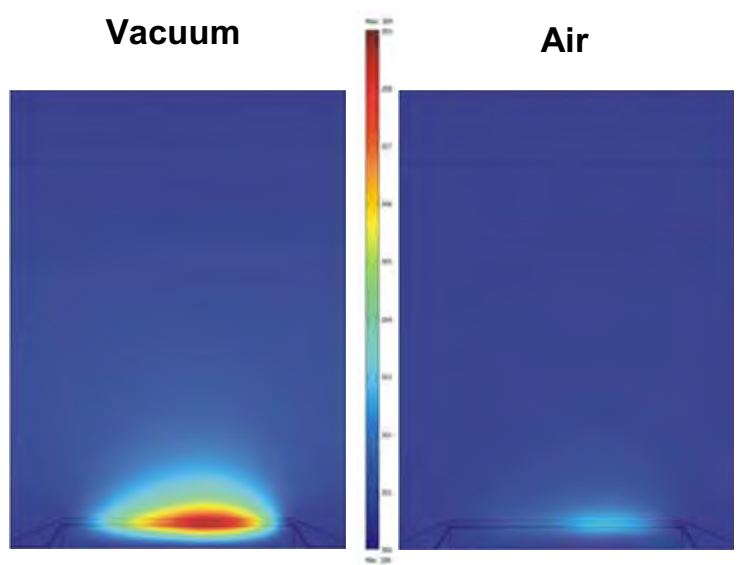

Figure 2: Steady-state temperature distribution of a symmetric BiSb / Sb - thermocouple air-bridge structure in air and vacuum. In both cases, the load power is $7.5 \mu \mathrm{W}$

with $\mathrm{k}_{\mathrm{B}}$ being the Boltzmann constant. The NEP due to the Johnson noise is

$$
\begin{aligned}
& N E P_{J}^{2}=\frac{4 k_{B} T \cdot R}{[\mid S]^{2}} \\
& =N E P_{T}^{2} \frac{1}{M}\left[1+\omega^{2} \tau^{2}\right]
\end{aligned}
$$

with $\mathrm{M}$ being the dimensionless figure of merit.

$$
M=\frac{\alpha^{2} \cdot T}{G \cdot R}
$$

With that, the modeled structure has an NEP of $16 \mathrm{pW} / \mathrm{Hz}^{1 / 2}$ in vacuum.

\section{Fabrication}

The manufacturing process of the thermocouple air-bridge structure is based on surface micromachining technology. A silicon substrate with a high resistivity and a thermal silicon dioxide layer onto both sides is used as starting basis. First, a $2.5 \mu \mathrm{m}$ thin polyimide layer was deposited as a sacrificial layer. After patterning the polyimide layer by using RIE oxygen plasma process, the polyimide is covered with a $200 \mathrm{~nm}$ PECVD nitride layer which serves as supporting membrane and insulates both the thermocouple and the substrate. The intrinsic tensile stress of the layer was $40 \mathrm{MPa}$ so as to avoid sticking effects. This layer was deposited by PECVD. The process parameters for the deposition of such $\mathrm{Si}_{\mathrm{x}} \mathrm{N}_{\mathrm{y}}$ membranes were studied in previous investigations [8]. Due to its high electrical and thermal conductivity, a $130 \mathrm{~nm}$ thick gold layer 
was used for the antenna structure, the wiring as well as the bond pads. The gold layer was deposited by electron beam evaporation and structured by lift-off. During the fabrication process an intermetallic reaction between the gold and the thermoelectric layers must be prevented. For that, a nickel diffusion barrier layer was used. Moreover, nickel was also applied to improve the electrical connection between the thermoelectric materials. The nickel layer was deposited by electron beam evaporation and structured by lift-off. For the thermoelectric sensing elements a material combination of n-type bismuth-antimony alloy and p-type antimony was chosen. The Seebeck coefficient $\alpha$ of these thin films is $\alpha=-100 \mu \mathrm{V} / \mathrm{K}$ for $\mathrm{BiSb}$ [9] and $35 \mu \mathrm{V} / \mathrm{K}$ for $\mathrm{Sb}$ [10].

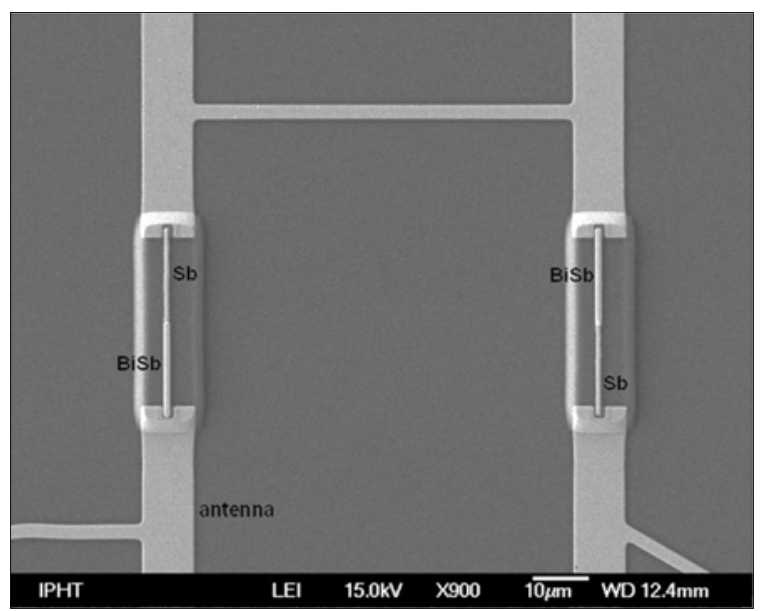

Figure 3: Scanning Electron Microscope (SEM) image of two antenna-coupled thermocouples.

Two completed thermocouples connected to an antenna are shown in Fig. 3. For the optimization of thermocouples the thermoelectric figure of merit $Z=\alpha^{2} \sigma / \lambda \quad(\alpha$ : the absolute Seebeck coefficient of the material, $\sigma$ : the electrical conductivity and $\lambda$ : the thermal conductivity) of each material, as well as the particular n-type/ptype material combination, are the most important material parameters. To increase the detectivity of the thermocouple, an optimized alloy $\mathrm{Bi}_{0.87} \mathrm{Sb}_{0.13}$ with a thermoelectric figure of merit of $\mathrm{Z}=0.5 \times 10^{-3} \mathrm{~K}^{-1}$ [11] was used as n-type material. The $150 \mathrm{~nm}$ thick Sb-layer as well as the $350 \mathrm{~nm}$ thick BiSb-layer were deposited at room temperature and structured via lift-off technique. To realize a high-quality electrical contact between nickel and the deposited thermoelectric materials, a dry etching step with argon ions (IBE) was accomplished before the deposition. The IBE step, as well as the following deposition process, were realized seamlessly under vacuum conditions. In order to optimize the sensor performance, the resistance of the thermocouples, which acts as a load resistance, was matched to the antenna. The resistance of a single thermocouple was in the range of $250 \Omega$ to
$350 \Omega$. After fabrication of the thermocouples, the sensing elements were passivated by $\mathrm{SiO}_{2}$. Since a low thermal mass reduces the thermal time constant, only a very thin $\mathrm{SiO}_{2}$ layer was deposited by atomic layer deposition (ALD). Using this technique, the structures were covered by a thickness of only $25 \mathrm{~nm}$. At the end of the fabrication process the carrier membrane as well as the passivation layer were structured against a resist mask by using an ICP-RIE process. After that the polyimide sacrificial layer was removed completely by using an isotropic RIE oxygen process. Thereby, very thin and mechanically stable freestanding air-bridges with the thermocouples on top were obtained, cf. Fig. 4.

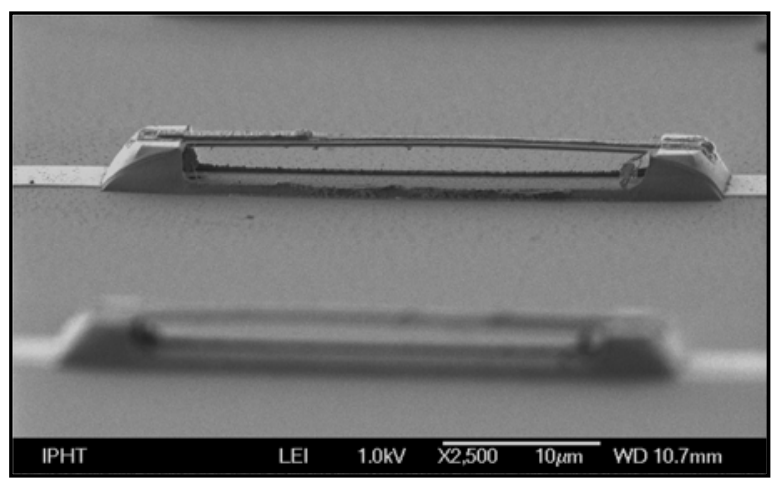

Figure 4: SEM image of the Thermocouple air-bridge structure after removing the sacrificial layer. The length of the obtained air-bridge structure is $30 \mu \mathrm{m}$ and the width is $3.5 \mu \mathrm{m}$.

\section{Results}

For the characterization of the air bridge structure an AC-DC-measurement method was used. In Fig. 5 the corresponding electrical circuit is shown. The thermocouple was loaded with an alternating current signal with a frequency of $100 \mathrm{kHz}$, whereas the resulting AC-voltage across the thermocouple and a $1000 \Omega$ resistor was measured. By ohm's law, it is possible to calculate the current through the resistor. The

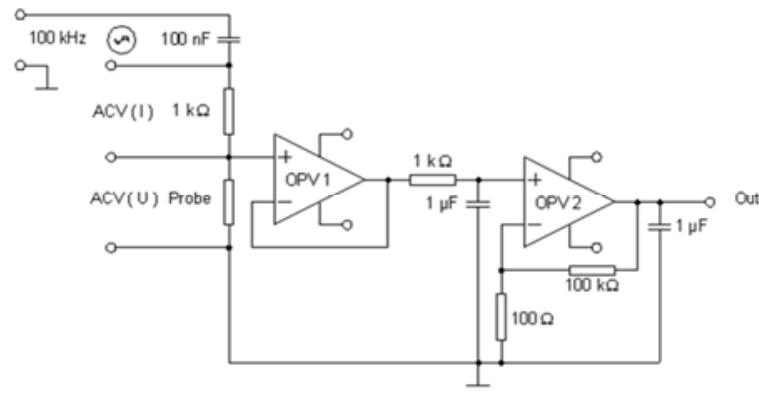

Figure 5: Circuit diagram of the AC-DC measurement method used to characterize the air bridge structure.

thermocouple is connected in series with the resistor; consequently, the current through the thermocouple is the same as through the resistor. The electrical power is dissipated within the thermocouple, resulting in a temperature gradient. Thus, a thermoelectric DC-voltage is 
generated, which is proportional to the electrical power. The thermoelectric DC-voltage was separated from the AC-voltage by an operational amplifier (op-amp). A second op-amp amplified the signal by a factor of 1000 . The measurement was accomplished under atmospheric as well as vacuum conditions. The results are summarized in Tab. 2. With the measured vacuum responsivity of $120 \mathrm{~V} / \mathrm{W}$ and a resistance of $290 \Omega$, an electrical NEP of $18 \mathrm{pW} / \mathrm{Hz}^{1 / 2}$ was calculated at room temperature. Note that the optical NEP, which includes the optical coupling efficiency, is higher than the electrical NEP since the coupling efficiency of the antenna is definitely less than 1.The measured responsivity under atmospheric conditions is about $33 \mathrm{~V} / \mathrm{W}$ which results in a vacuum factor of 3.6. The measurement of the time constant of the airbridge structure yielded $19 \mu \mathrm{s}$ under atmospheric pressure [12]. Summary, the simulations and the measurements for a single element are in good agreement.

Table 2: Results of the measurements for vacuum and air conditions.

\begin{tabular}{|l|l|}
\hline Physical parameter & \multicolumn{1}{|c|}{$\begin{array}{c}\text { Results of the } \\
\text { measurements }\end{array}$} \\
\hline Responsivity (vac.) & $120 \mathrm{~V} / \mathrm{W}$ \\
\hline Responsivity (air) & $33 \mathrm{~V} / \mathrm{W}$ \\
\hline Time constant (air) & $19 \mu \mathrm{s}$ \\
\hline Vacuum factor & 3.6 \\
\hline NEP (vac.) & $18 \mathrm{pW} / \mathrm{Hz}^{1 / 2}$ \\
\hline NEP (air) & $67 \mathrm{pW} / \mathrm{Hz}^{1 / 2}$ \\
\hline
\end{tabular}

\section{Conclusion}

We have presented an air-bridge structure supporting a thermocouple-based conducting strip acting as both Joule heater and temperature sensor for an uncooled antenna coupled thermal sensor. In the first step the air-bridge structure was simulated to find suitable parameters. The air-bridge was fabricated by surface micromachining. As sacrificial layer polyimide was used and covered with a $200 \mathrm{~nm}$ thin SiN as supporting layer. On this layer, the thermocouple was patterned and covered with a $25 \mathrm{~nm}$ thin ALD-SiN layer. The characterization of this air bridge structure was performed with an adapted AC-DC-measurement method. The measured results are in accordance with the simulation. The measured vacuum responsivity is $120 \mathrm{~V} / \mathrm{W}$ and the vacuum NEP for a single element is $18 \mathrm{pW} / \mathrm{Hz}^{1 / 2}$. As a next step, further dimension optimization of the air bridge will be performed to achieve lower thermal conductance and.

\section{References}

[1] A. Ihring, E. Kessler, U. Dillner, F. Haenschke, U. Schinkel, M. Schubert, R. Haehle, H.-G. Meyer, High performance uncooled $\mathrm{THz}$ sensing structures based on antenna-coupled air-bridges, MEE 98 (2012) 512-515.

[2] A. Luukanen, R.H. Hadfield, A.J. Miller, E.N. Grossman, A superconducting antenna-coupled microbolometer for $\mathrm{THz}$ applications, Proc. SPIE 5411 (2004) 121-126.

[3] J.P. Rice, E.N. Grossman, D.A. Rudman, Antenna-coupled high- $T_{C}$ air-bridge microbolometer on silicon, Appl. Phys. Lett. 65 (1994) 773-775.

[4] A.J. Miller, A. Luukanen, E.N. Grossman, Micromachined antenna-coupled microbolometers for terahertz imaging arrays, Proc. SPIE 5411 (2004) 18-24.

[5] J.A. Cox, R. Higashi, F. Nusseibeh, K. Newstrom-Peitso, C. Zins, R. Osiander, J. Lehtonen, E. Dodson, Uncooled MEMS-based detector arrays for $\mathrm{THz}$ imaging applications, Proc. SPIE 7311 (2009) 73110R 1-11.

[6] E. Peytavit, P. Agnèse, J-L. Ouvrier Buffet, A. Beguin, F. Simoens, Room temperature terahertz microbolometers, Proc. IRMMW-THz Conf. (2005) 257258.

[7] R.A. Smith, F.E. Jones, R.P. Chasmar, The Detection and Measurement of Infra-red Radiation, Oxford, Clarendon Press (1968)

[8] A. Ihring, E. Kessler, U. Dillner, F. Haenschke, U. Schinkel, H.-G. Meyer, Surface-micromachined thermoelectric infrared focal-plane array with high detectivity for room temperature operation, MEE. 88 (2011) 2267-2271.

[9] F. Voelklein, E. Kessler, Thermoelectric properties of $\mathrm{Bi}_{1-\mathrm{x}} \mathrm{Sb}_{\mathrm{x}}$ films with $0<\mathrm{x} \leq 0.3$, Thin Solid Films, 155 (1987) 197-208.

[10] F. Voelklein, E. Kessler, Thermal Conductivity and Thermoelectric Figure of Merit of Thin Antimony Films, Phys. Status Solidi B, 158 (1990) 521-529.

[11] F. Voelklein, E. Kessler, Thermal Conductivity and Thermoelectric Figure of Merit of $\mathrm{Bi}_{1-x} \mathrm{Sb}_{x}$ Films with $0<\mathrm{x} \leqq 0.3$, Phys. Status Solidi B, 143 (1987) 121130.

[12] A.K. Huhn, G. Spickermann, A. Ihring, U. Schinkel, H.-G. Meyer, P. Haring Bolivar, Fast antenna-coupled terahertz based on uncooled thermo elements, Proc. IRMMW-THz Conf. (2012). 